\title{
ON THE VOLUME OF THE INTERSECTION OF TWO $L_{p}^{n}$ BALLS
}

\author{
G. SCHECHTMAN AND J. ZINN
}

(Communicated by William J. Davis)

\begin{abstract}
In this note we prove that the proportion of the volume left in the $L_{p}^{n}$ ball after removing a $t$-multiple of the $L_{q}^{n}$ ball is of order $e^{-c t^{p} n^{p / q}}$.
\end{abstract}

\section{INTRODUCTION}

This note deals with the following problem, the case $p=1, q=2$ of which was raised by Vitali Milman: What is the volume left in the $L_{p}^{n}$ ball after removing a $t$-multiple of the $L_{q}^{n}$ ball? Recall that the $L_{r}^{n}$. ball is the set $\left\{\left(t_{1}, t_{2}, \ldots, t_{n}\right) ; t_{i} \in \mathbf{R}, n^{-1} \sum_{i=1}^{n}\left|t_{i}\right|^{r} \leq 1\right\}$ and note that for $0<p<q<\infty$ the $L_{q}^{n}$ ball is contained in the $L_{p}^{n}$ ball.

In Corollary 4 below, we show that after normalizing Lebesgue measure so that the volume of the $L_{p}^{n}$ ball is one, the answer to the problem above is of order $e^{-c t^{p} n^{p / q}}$ for $T<t<\frac{1}{2} n^{1 / p-1 / q}$, where $c$ and $T$ depend on $p$ and $q$ but not on $n$.

The main theorem, Theorem 3, deals with the corresponding question for a certain measure on the $L_{p}^{n}$ sphere. Theorem 3 and Corollary 4 , together with some other remarks, form $\S 3$. In $\S 2$ we introduce a class of random variables to be used in proving the main theorem. These random variables are related to $L_{p}$ in the same way that Gaussian variables are related to $L_{2}$.

\section{Preliminaries}

Here we introduce a class of random variables to be used in the proof of the main theorem, and we summarize some of their properties. Fix a $0<p<\infty$ and let $x, x_{1}, x_{2}, \ldots, x_{n}$ be independent random variables each with density function $c_{p} e^{-t^{p}}, t>0$. Note that necessarily $c_{p}=p / \Gamma(1 / p)$. Let $\Delta_{p}$ denote

Received by the editors September 14, 1989.

1980 Mathematics Subject Classification (1985 Revision). Primary 52A20; Secondary 60E15.

The work of the first author was partly supported by the US-Israel BSF and by the Glikson Foundation.

The work of the second author was partly supported by NSF DMS-86-01250 and by the Texas Advanced Research Program, Grant no. 3825. 
the positive quadrant of the sphere of $l_{p}^{n}$, i.e.,

$$
\Delta_{p}=\left\{\left(t_{1}, t_{2}, \ldots, t_{n}\right) ; t_{i} \geq 0,\|t\|_{p}^{p}=: \sum t_{i}^{p}=1\right\},
$$

and for, $\lambda$, Lebesgue measure on $\mathbf{R}^{n}$, let $\bar{\mu}$ be the Borel probability on $\Delta_{p}$ given by

$$
\bar{\mu}(A)=\frac{\lambda\left(\left\{t \in \mathbf{R}_{+}^{n}: \frac{t}{\|t\|_{p}} \in A, \quad\|t\|_{p} \leq 1\right\}\right)}{\lambda\left(\left\{t \in \mathbf{R}_{+}^{n}:\|t\|_{p} \leq 1\right\}\right)} .
$$

Notice that for all $\varepsilon>0$,

$$
\bar{\mu}(A)=\frac{\lambda\left(\left\{\left(t_{1}, \ldots, t_{n}\right) \in \mathbf{R}_{+} \cdot A: a-\varepsilon<\|t\|_{p} \leq a+\varepsilon\right\}\right)}{\lambda\left(\left\{\left(t_{1}, \ldots, t_{n}\right) \in \mathbf{R}_{+}^{n}: a-\varepsilon<\|t\|_{p} \leq a+\varepsilon\right\}\right)} .
$$

Note that for $p=1$ and $p=2 \bar{\mu}$ is the normalized surface measure on $\Delta_{p}$, and that for all $p$,

$$
\bar{\nu}(A)=n \int_{0}^{1} r^{n-1} \bar{\mu}\left(\frac{A}{r}\right) d r
$$

where $\bar{\nu}$ denotes the normalized Lebesgue measure on the positive quadrant of the $l_{p}^{n}$ ball. The first claim is known, though we could not locate a reference.

Lemma 1. Put $S=\left(\sum_{i=1}^{n} x_{i}^{p}\right)^{1 / p}$; then $\left(\frac{x_{1}}{S}, \frac{x_{2}}{S}, \ldots, \frac{x_{n}}{S}\right)$ induces the measure $\bar{\mu}$ over the positive quadrant of the sphere of $l_{p}^{n}$. Moreover, $\left(\frac{x_{1}}{S}, \frac{x_{2}}{S}, \ldots, \frac{x_{n}}{S}\right)$ is independent of $S$.

Proof. For any Borel subset $A$ of $\Delta_{p}$,

$$
\begin{aligned}
& P\left(\left(\frac{x_{1}}{S}, \frac{x_{2}}{S}, \ldots, \frac{x_{n}}{S}\right) \in A \mid S=a\right) \\
& =\lim _{\varepsilon \rightarrow 0} \frac{P\left(\left(x_{1}, \ldots, x_{n}\right) \in \mathbf{R}_{+} A \& a-\varepsilon \leq S \leq a+\varepsilon\right)}{P(a-\varepsilon \leq S \leq a+\varepsilon)} \\
& =\lim _{\varepsilon \rightarrow 0} \int_{\substack{\left(t_{1}, \ldots, t_{n}\right) \in \mathbf{R}_{+} A \\
(a-\varepsilon)^{p}<\sum t_{i}^{p}<(a+\varepsilon)^{p}}} e^{-\sum t_{i}^{p}} d t / \int_{\substack{\left(t_{1}, \ldots, t_{n}\right) \in \mathbf{R}_{+}^{n} \\
(a-\varepsilon)^{p}<\sum t_{i}^{p}<(a+\varepsilon)^{p}}} e^{-\sum t_{i}^{p}} d t \\
& \leq \limsup _{\varepsilon \rightarrow 0} e^{-(a-\varepsilon)^{p}+(a-\varepsilon)^{p}} \int_{\substack{\left(t_{1}, \ldots, t_{n}\right) \in \mathbf{R}_{+} A \\
(a-\varepsilon)^{p}<\sum t_{i}^{p}<(a+\varepsilon)^{p}}} d t / \int_{\substack{\left(t_{1}, \ldots, t_{n}\right) \in \mathbf{R}_{+}^{n} \\
(a-\varepsilon)^{p}<\sum t_{i}^{p}<(a+\varepsilon)^{p}}} d t \\
& =\bar{\mu}(A) \text {. }
\end{aligned}
$$

Similarly,

$$
P\left(\left(\frac{x_{1}}{S}, \frac{x_{2}}{S}, \ldots, \frac{x_{n}}{S}\right) \in A \mid S=a\right) \geq \bar{\mu}(A) .
$$

This proves that $P\left(\left(\frac{x_{1}}{S}, \frac{x_{2}}{S}, \ldots, \frac{x_{n}}{S}\right) \in A\right)=\bar{\mu}(A)$ and that $\left(\frac{x_{1}}{S}, \frac{x_{2}}{S}, \ldots, \frac{x_{n}}{S}\right)$ is independent of $S$.

In the next claim we gather some more properties of the random variables $x_{i}$. 
Lemma 2. Let $x, x_{1}, \ldots, x_{n}$ be as above; then

1. $c_{p}$ is bounded away from zero and infinity when $p \rightarrow \infty$.

2. For all $h>0$ and all $0<p<\infty, \mathbf{E} e^{-h x^{p}}=\left(\frac{1}{1+h}\right)^{1 / p}$. In particular, $\mathbf{E} e^{-h x^{p}} \geq e^{-h / p}$ for all $h>0$ and $\mathbf{E} e^{-h x^{p}} \leq e^{-h / 2 p}$ for all $0<h \leq 1$.

3. For all $0<u<\infty$ and all $0<p<\infty, P\left(x^{p}>u\right) \geq \frac{c_{p}}{2 p} e^{-2 u}$. If $p \geq 1$ and $u \geq 1$, then also $P\left(x^{p}>u\right) \leq \frac{c_{p}}{p} e^{-u / 2}$. In particular, for $p \geq 1$ and all $u, P\left(x^{p}>u\right) \leq C e^{-u / 2}$ for some universal $C$.

4. For all $1 \leq p \leq q<\infty$, if $n$ is large enough, then $\mathbf{E}\left(\sum_{i=1}^{n} x_{i}^{q}\right)^{1 / q}$ is equivalent, with universal constants, to $q^{1 / p} n^{1 / p}$, if $q \leq \log n$, and to $(\log n)^{1 / p}$, otherwise.

Proof.

(1) follows easily from the fact that $c_{p}=p / \Gamma(1 / p)=\Gamma\left(\frac{1}{p}+1\right)^{-1}$.

(2) is a simple computation.

(3) is also simple; here is a sketch of the proof.

$$
\begin{aligned}
P\left(x^{p}>u\right) & =c_{p} \int_{u^{1 / p}}^{\infty} e^{-t^{p}} d t \\
& \geq c_{p} \int_{u^{1 / p}}^{(u+1)^{1 / p}} \frac{p t^{(p-1)}}{p(u+1)^{(p-1) / p}} e^{-t^{p}} d t \\
& =\frac{c_{p}}{p(u+1)^{(p-1) / p}}\left(1-\frac{1}{e}\right) e^{-u} \\
& \geq \frac{c_{p}}{2 p(u+1)} e^{-u} \\
& \geq \frac{c_{p}}{2 p} e^{-2 u} .
\end{aligned}
$$

The other inequality in (3) is proved in a similar way.

(4) First note that for all $0<p, q<\infty$

$$
\mathbf{E} x^{q}=c_{p} \int_{0}^{\infty} t^{q} e^{-t^{p}} d t=\frac{c_{p}}{p} \Gamma\left(\frac{q+1}{p}\right) ;
$$

so that, by Holder's inequality and 1 above, if $1 \leq p \leq q<\infty$,

$$
\mathbf{E}\left(\sum_{i=1}^{n} x_{i}^{q}\right)^{1 / q} \leq\left(\sum_{i=1}^{n} \mathbf{E} x_{i}^{q}\right)^{1 / q}=\left(\frac{c_{p}}{p} \Gamma\left(\frac{q+1}{p}\right)\right)^{1 / q} n^{1 / q} \leq C q^{1 / p} n^{1 / q}
$$

for some universal $C$. For the lower bound, note first that, by (3) above,

$$
P\left(\max _{1 \leq i \leq n} x_{i}>t\right) \geq 1-\left(1-\frac{c_{p}}{2 p} e^{-2 t^{p}}\right)^{n} \text {. }
$$

For $n \geq 20 p / c_{p}$, choose $t=2^{1 / p}\left(\log \frac{n c_{p}}{2 p}\right)^{1 / p}$ to get that, for some universal $c$,

$$
P\left(\max _{1 \leq i \leq n} x_{i}>c(\log n)^{1 / p}\right) \geq 1 / 2 \text {. }
$$


In particular, $\mathbf{E} \max _{1 \leq i \leq n} x_{i} \geq c(\log n)^{1 / p}$. This already implies the claim if $q>$ $\log n$, since in this case $\left(\sum_{i=1}^{n} x_{i}^{q}\right)^{1 / q}$ is universally equivalent to $\max _{1 \leq i \leq n} x_{i}$. If $q \leq \log n$, divide $\{1,2, \ldots, n\}$ into approximately $n / e^{q}$ disjoint sets of cardinality approximately $e^{q}$ each; then

$$
\begin{aligned}
\mathbf{E}\left(\sum_{i=1}^{n} x_{i}^{q}\right)^{1 / q} & =\mathbf{E}\left(\sum_{j}\left(\sum_{i \in \sigma_{j}} x_{i}^{q}\right)^{q / q}\right)^{1 / q} \\
& \geq \mathbf{E}\left(\sum_{j}\left(\max _{i \in \sigma_{j}} x_{i}\right)^{q}\right)^{1 / q} \\
& \geq\left(\sum_{j}\left(\mathbf{E} \max _{i \in \sigma_{j}} x_{i}\right)^{q}\right)^{1 / q} \\
& \geq c^{\prime}\left(\log e^{q}\right)^{1 / p}\left(n / e^{q}\right)^{1 / q} \\
& \geq c^{\prime \prime} q^{1 / p} n^{1 / q} .
\end{aligned}
$$

The statement in Lemma 2.4, for the case $p=2$, was noticed by the firstnamed author several years ago while seeking a precise estimate for the dimension of the Euclidean sections of $l_{p}^{n}$ spaces (see [MS] p. 145, Remark 4.5). The original proof was more complicated. The proof presented here is an adaptation of a proof of the case $p=2$ shown to us by J. Bourgain.

\section{The MAIN RESULT}

Theorem 3. For all $1 \leq p<q<\infty$ there are constants $c=c(p, q), C=$ $C(p, q)$, and $T=T(p, q)$ such that if $\mu$ denotes the probability measure on the positive quadrant of the unit sphere of $L_{p}^{n}$ given by $\mu(A)=\bar{\mu}\left(n^{1 / p} A\right)$, then

$$
\mu\left(\|u\|_{L_{q}^{n}}>t\right) \leq \exp \left(-c t^{p} n^{p / q}\right)
$$

for all $t>T$, and

$$
\mu\left(\|u\|_{L_{q}^{n}}>t\right) \geq \exp \left(-C t^{p} n^{p / q}\right)
$$

for all $2 \leq t \leq \frac{1}{2} n^{1 / p-1 / q}$.

Moreover, for $q>2 p$ (or any other universal positive multiple of $p$ ), one can take $c(p, q)=\frac{\gamma}{p}, C(p, q)=\frac{\Gamma}{p}$, and $T(p, q)=\tau \min \{q, \log n\}^{1 / p} \leq q^{1 / p}$. Here $\gamma, \Gamma$, and $\tau$ are universal constants.

Proof. By Lemma 1 above,

$$
\mu\left(\|u\|_{L_{q}^{n}}>t\right)=P\left(n^{1 / p-1 / q}\left(\sum_{i=1}^{n} x_{i}^{q}\right)^{1 / q} /\left(\sum_{i=1}^{n} x_{i}^{p}\right)^{1 / p}>t\right),
$$


where $x_{i}$ are independent random variables each with density $c_{p} e^{-t^{p}}$. Assume, for the simplicity of the presentation, that $n$ is even. Put $S=\left(\sum_{i=1}^{n} x_{i}^{p}\right)^{1 / p}$ and let $p_{j}, j=1,2, \ldots, n / 2$, be positive numbers with sum $\leq 1 / 2$. Then

$$
\begin{aligned}
& P\left(n^{1 / p-1 / q}\left(\sum_{i=1}^{n} x_{i}^{q}\right)^{1 / q} /\left(\sum_{i=1}^{n} x_{i}^{p}\right)^{1 / p}>t\right) \\
& \quad=P\left(\sum_{i=1}^{n} x_{i}^{q}>\frac{t^{q}\left(\sum_{i=1}^{n} x_{i}^{p}\right)^{q / p}}{n^{q / p-1}}\right) \\
& \quad \leq \sum_{i=1}^{n / 2} P\left(x_{i}^{*}>t p_{i}^{1 / q} S / n^{1 / p-1 / q}\right)+P\left(\sum_{i=n / 2+1}^{n} x_{i}^{* q}>t^{q} S^{q} / 2 n^{q / p-1}\right),
\end{aligned}
$$

where $\left\{x_{j}^{*}\right\}$ denotes the nonincreasing rearrangement of $\left\{\left|x_{j}\right|\right\}$.

Since

$$
\begin{aligned}
\sum_{j=n / 2+1}^{n} x_{i}^{* q} & \leq \frac{n}{2} x_{n / 2}^{* q} \leq \frac{n}{2}\left(\frac{2}{n} \sum_{i=1}^{n / 2} x_{i}^{* p}\right)^{q / p} \\
& \leq 2^{q / p-1} S^{q} / n^{q / p-1},
\end{aligned}
$$

we get that, if $t \geq 2^{1 / p}$, the second term in (3) is zero.

To evaluate the first term in (3), fix $1 \leq j \leq n / 2$. Then,

$$
\begin{aligned}
P\left(x_{j}^{*}>t p_{j}^{1 / q} S / n^{1 / p-1 / q}\right) & \leq\left(\begin{array}{c}
n \\
j
\end{array}\right) P\left(x_{1}, \ldots, x_{j}>t p_{j}^{1 / q} S / n^{1 / p-1 / q}\right) \\
& \leq\left(\begin{array}{c}
n \\
j
\end{array}\right) P\left(x_{1}^{p}, \ldots, x_{j}^{p}>t^{p} p_{j}^{p / q} \sum_{i=j+1}^{n} x_{i}^{p} / n^{1-p / q}\right) .
\end{aligned}
$$

From Lemma 2.3 and 2.2 we get that the last expression is dominated by

$$
\begin{aligned}
&\left(\begin{array}{c}
n \\
j
\end{array}\right) C^{j} \mathbf{E} \exp \left(-j p_{j}^{p / q} t^{p} \sum_{i=j+1}^{n} x_{i}^{p} / n^{1-p / q}\right) \\
& \leq\left(\begin{array}{c}
n \\
j
\end{array}\right) C^{j} \exp \left(-j p_{j}^{p / q} t^{p}(n-j) / 2 p n^{1-p / q}\right)
\end{aligned}
$$

for some universal $C$. Note that the last inequality holds if $j n^{p / q-1} p_{j}^{p / q} t^{p} \leq 1$. If this is not the case, the probability we are trying to evaluate is zero. Finally, the last term is dominated by

$$
\exp \left(j\left(\log \frac{e n}{j}+C-\frac{p_{j}^{p / q} t^{p} n^{p / q}}{4 p}\right)\right) .
$$

Now, for $\alpha$ to be chosen momentarily, let $p_{j}, j=1, \ldots, n / 2$, be such that

$$
j\left(\log \frac{e n}{j}+C-\frac{p_{j}^{p / q} t^{p} n^{p / q}}{4 p}\right)=-\alpha n^{p / q} t^{p},
$$


that is,

$$
p_{j}=\left(4 p \frac{\log \frac{e n}{j}}{t^{p} n^{p / q}}+\frac{4 C p}{t^{p} n^{p / q}}+\alpha \frac{4 p}{j}\right)^{q / p}
$$

We thus get that, for some universal constant $C$,

$$
p_{j} \leq 2^{q / p-1} \frac{(C p)^{q / p}\left(\log \frac{e n}{j}\right)^{q / p}}{t^{q} n}+2^{q / p-1} \alpha^{q / p} \frac{(4 p)^{q / p}}{j^{q / p}}
$$

It is easy to see that, for $1 \leq p<q<\infty$,

$$
\sum_{j=1}^{n / 2}\left(\log \frac{e n}{j}\right)^{q / p} \leq A n \min \left\{n q^{q / p},(\log n)^{q / p}\right\}
$$

for some universal $A$. Thus the sum over the first terms in (5) is smaller than $1 / 4$ if, for some universal $\gamma, t>\gamma \min \left\{q^{1 / p},(\log n)^{1 / p}\right\}$. The second term is bounded by $1 / 4$ if $\alpha<B \frac{1}{p}\left(\frac{q}{p}-1\right)^{p / q}$, for some universal $B$. Choosing $\alpha$ to satisfy this inequality and using (3), (4), and (5) we get that, for $t>$ $\gamma \min \left\{q^{1 / p},(\log n)^{1 / p}\right\}$,

$$
\mu\left(\|u\|_{L_{q}^{n}}>t\right) \leq \frac{n}{2} e^{-\alpha n^{p / q} t^{p}}
$$

Under the conditions on $t$, the factor $n / 2$ can be absorbed in the second term (changing $\alpha$ to another constant of the same order of magnitude as a function of $p$ ), thus proving (1).

We now turn to the proof of the lower bound (2), which is simpler. Using Lemma 1 again,

$$
\begin{aligned}
\mu\left(\|u\|_{L_{q}^{n}}>t\right) & =P\left(n^{1 / p-1 / q}\left(\sum_{i=1}^{n} x_{i}^{q}\right)^{1 / q} /\left(\sum_{i=1}^{n} x_{i}^{p}\right)^{1 / p}>t\right) \\
& \geq P\left(x_{1}>S t / n^{1 / p-1 / q}\right) \\
& =P\left(x_{1}>\frac{t}{\left(n^{(1-p / q)}-t^{p}\right)^{1 / p}}\left(\sum_{i=2}^{n} x_{i}^{p}\right)^{1 / p}\right) .
\end{aligned}
$$

Since $t^{p} \leq \frac{1}{2} n^{(1-p / q)}$, this dominates

$$
P\left(x_{1}>\frac{2^{1 / p} t}{n^{1 / p-1 / q}}\left(\sum_{i=2}^{n} x_{i}^{p}\right)^{1 / p}\right)
$$


Now, by Lemma 2.3,

$$
\begin{aligned}
P\left(x_{1}>\frac{2^{1 / p} t}{n^{1 / p-1 / q}}\left(\sum_{i=2}^{n} x_{i}^{p}\right)^{1 / p}\right) & \geq \frac{c_{p}}{2 p} \mathbf{E} \exp \left(-4 t^{p} \sum_{i=2}^{n} x_{i}^{p} / n^{(1-p / q)}\right) \\
& =\frac{c_{p}}{2 p}\left(\mathbf{E} \exp \left(-4 t^{p} x_{1}^{p} / n^{(1-p / q)}\right)\right)^{n-1} \\
& =\frac{c_{p}}{2 p}\left(\frac{1}{1+\frac{4 t^{p}}{n^{1-p / q}}}\right)^{(n-1) / p} \quad \text { (by Lemma 2.2) } \\
& \geq \frac{c_{p}}{2 p} \exp \left(-\frac{4 t^{p}(n-1)}{p n^{(1-p / q)}}\right) \\
& \geq \frac{c_{p}}{2 p} e^{4 t^{p} n^{p / q} / p} .
\end{aligned}
$$

Finally observe that, since $c_{p}$ is bounded away from zero and $t \geq 2$, the factor $\frac{c_{p}}{2 p}$ can be absorbed in the second term (changing 4 to another universal constant).

Remarks. 1. It follows from the proof that, for $n$ large enough and $q$ close to $p$, one can take $c(p, q)=\frac{c}{p}\left(\frac{q}{p}-1\right)$ for some universal constant $c$.

2. It follows from the statement of the theorem that, for $q=\infty$,

$$
\mu\left(\|u\|_{\infty}>t\right) \leq e^{-\gamma t^{p} / p}
$$

for all $t>\tau(\log n)^{1 / p}$, and

$$
\mu\left(\|u\|_{\infty}>t\right) \geq e^{-\Gamma t^{p} / p}
$$

for all $2 \leq t \leq \frac{1}{2} n^{1 / p}$, where $\gamma, \Gamma$, and $\tau$ are universal constants.

3. Note that it follows from Lemma 1 and Lemma 2.4 that the order of magnitude of $T$ is the correct one.

4. The restriction $p \geq 1$ in Theorem 3 above and in Corollary 4 below can be replaced by $p>0$ if one replaces the inequality $t \geq 2$ with $t \geq d$, for some $d$ depending only on $p$ and $q$, and if one removes the "moreover" part. We didn't check the dependence of the constants on $p$ and $q$ in this case.

5. The measure $\mu$ and the normalized Lebesgue measure are equivalent with constant at most $n^{1 / 2}$. It follows that a similar statement holds also for the normalized Lebesgue measure on the positive quadrant of the unit sphere of $L_{p}^{n}$. For $p=1$ and $p=2$ the measures $\bar{\mu}$ and normalized Lebesgue measure on the positive quadrant of the $L_{p}^{n}$ sphere are equal.

Our last remark is that one can get a similar statement for the full balls. We state it as a corollary. 
Corollary 4. For all $1 \leq p<q<\infty$, there are constants $c=c(p, q), C=$ $C(p, q)$, and $T=T(p, q)$ such that if $\nu$ denotes the normalized Lebesgue measure on the ball of $L_{p}^{n}$, then for all $n$ large enough,

$$
\nu\left(\|u\|_{L_{q}^{n}}>t\right) \leq \exp \left(-c t^{p} n^{p / q}\right)
$$

for all $t>T$, and

$$
\nu\left(\|u\|_{L_{q}^{n}}>t\right) \geq \exp \left(-C t^{p} n^{p / q}\right)
$$

for all $2 \leq t \leq \frac{1}{2} n^{1 / p-1 / q}$. Moreover, for $q>2 p$ (or any other universal positive multiple of $p)$, one can take $c(p, q)=\frac{\gamma}{p}, C(p, q)=\frac{\Gamma}{p}$, and $T(p, q)=$ $\tau \min \{q, \log n\}^{1 / p} \leq q^{1 / p}$, where $\gamma, \Gamma$, and $\tau$ are universal constants.

The proof follows easily from Theorem 3 and the formula

$$
\nu(A)=n \int_{0}^{1} r^{n-1} \mu\left(\frac{A}{r}\right) d r
$$

which holds for all Borel sets $A$ in the ball of $L_{p}^{n}$.

\section{REFERENCES}

[MS] V. D. Milman and G. Schechtman, Asymptotic theory of finite dimensional normed spaces, Lecture Notes in Math., vol. 1200, Springer-Verlag, New York, 1986.

Department of Theoretical Mathematics, The Weizmann Institute of Science, ReHOVOT, ISRAEL

Department of Mathematics, Texas A\&M University, College Station, Texas 77843 